\title{
Analysis of the Dynamic Change of Vegetation Cover in the Mechanical Forest Field of Sehan Dam Based on RS
}

\author{
Yun Shang", Yanbin Qin, Pinyang Hu, Han Zheng, Zhi Zeng \\ Chengdu University of Technology, Chengdu, Sichuan 610059, China.
}

\begin{abstract}
In order to study the dynamic changes of vegetation cover in saihanba mechanical forest farm in the past 30 years, this paper used the Landsat remote sensing images of the area in 1989, 2001 and 2016 provided by the geospatial data cloud platform, and extracted the vegetation coverage information of saihanba mechanical forest farm with normalized vegetation index and pixel dichotomy, and analyzed the dynamic changes of vegetation cover in the three periods. Results show that fr om 1989 to 2001, mechanical sihanba forest vegetation cover age as a whole is in a state of slow wave growth, the western region is relatively stable, relatively obvious growth in central China, the eastern region has no obvious change. From 2001 to 2016, the FVC of this region has been significantly increased, especially in the western region where the FVC has been transformed from very low to very high FVC.The comprehensive results show that the vegetation cover of saihanba mechanical forest farm has undergone earth-shaking changes through artificial afforestation, creating a green miracle from vast wasteland to green water and green mountains, and bringing great ecological and social benefits to human beings.
\end{abstract}

Keywords: Saihanba Mechanical Forest Farm; Remote Sensing; NDVI; Vegetation Cover; Dynamic Change

\section{Introduction}

\subsection{The research background}

By chance, I watched the TV series The Most Beautiful Youth and got to know Saihanba, a place that creates green miracles. Once upon a time, it was a lush, lush forest, wild animals gathered in a paradise, is the emperor Mulan autumn hunting place. However, with the fall of the Qing Dynasty, Emperor Tongzhi issued a decree to open Saihanba for reclamation. Within a few years, the virgin forest had disappeared. Without the protection of forests and the fact that Saihanba is 1,500 meters above sea level, Beijing is only 40 meters above that kilometer, Beijing suffers from sandstorms almost every year. In order to keep the desert away from Beijing, it is necessary to store and preserve the water in Beijing and Tianjin while meeting the demand of the surrounding residents for wood. The national forestry ministry decided in the year to build a large mechanical forest farm in Saihanba.

\subsection{Research progress at home and abroad}

1.2.1 Research progress in China

Monitoring vegetation cover by remote sensing technology is a common technique used by Chinese scholars. Many researches and innovations have been made and some achievements have been made. For example, Jin Xiaomei et al. studied the change of vegetation coverage in Hedulan Area of Qaidam by estimating the vegetation coverage in the study area, and elucidated its influencing factors at the same time. Tian Shuai et al. made use of and classified the remote sensing images of Baoding, and obtained the characteristics of spatio-temporal transformation. These scholars obtained the change characteristics of vegetation cover in the study area through the study and analysis of the examples, which played a constructive role in the ecological environment governance.

1.2.2 Research progress abroad

Ground survey and remote sensing inversion are the main methods used by overseas scholars to measure vegetation coverage. The analysis of vegetation cover in the study area by remote sensing inversion technology has become the mainstream under the premise of the development of technology and the improvement of remote sensing estimation methods. For example, Stephens PR and other scholars calculated the vegetation coverage in the study area by studying the nonlinear empirical model between the vegetation index and. Tucker $\mathrm{C}$ et al. analyzed the vegetation in the Northern Hemisphere, and the study showed that the reason why the vegetation index performance in many regions of Eurasia kept increasing for several years was global warming.

\subsection{Research significance}

Based on remote sensing data, the vegetation coverage of Saihanba mechanical forest farm was analyzed and studied by using and pixel dichotomy method, and its changing trend was known, which provided a theoretical basis for the evaluation of

Copyright (C) 2020 Yun Shang et al.

doi: $10.18282 / 1-e . v 9 i 4.1714$

This is an open-access article distributed under the terms of the Creative Commons Attribution Non-Commercial License (http://creativecommons.org/licenses/by-nc/4.0/), which permits unrestricted non-commercial use, distribution, and reproduction in any medium, provided the original work is properly cited. 
environmental and ecological benefits in this area. Let this piece of green soil better benefit human beings, get the maximum degree of optimized use.

\subsection{Research methods}

There are many methods to monitor the dynamic changes of vegetation. In this paper, the Normalized Vegetation Index (NDVI) method is selected ${ }^{[1]}$. This method can not only effectively eliminate the influence caused by different terrain, but also reduce the interference of the atmosphere on monitoring. Its calculation formula is:

$$
N D V I=\frac{N I R-\operatorname{Re} d}{N I R+R e d}
$$

Wherein, Nir represents the reflection value in the near infrared band, and Red represents the reflection value in the Red band. The value range of NDVI is $-1 \sim 1$, so outliers should be removed. The characteristics of NDVI were as follows: vegetation coverage was positively correlated with NDVI; Vegetation NDVI $>0$; The NDVI value of construction land and bare land is near 0 . NDVI $<0$ is water body.

\section{Overview of the study area and data collection}

\subsection{Overview of the study area}

Saihanba Mechanical Forest Farm is located in Bashang District, north of Weichang Manchu and Mongolian Autonomous County, Chengde City, Hebei Province. The central location is $42^{\circ} 22^{\prime} 42.31^{\prime \prime} N 、 116^{\circ} 53^{\prime} 117.31^{\prime \prime} E$, The altitude is $1010-1939.9$ meter, The study area covers an area of square kilometers. The landform of this area is more complex, plateau, hills, floodplain, mountain interweave spread ${ }^{[2]}$. Saihanba mechanical forest farm has a high altitude, which determines that it is a typical semi-arid, semi-humid and extremely cold continental monsoon climate with extreme temperature, large temperature difference and annual average precipitation of millimeters.

\subsection{The data source}

The remote sensing data in this paper are downloaded through the geospatial data cloud platform ( http://www.gscloud.cn / ). Considering that the most prosperous season of vegetation growth is summer, the calculation of vegetation coverage will be more accurate, so the remote sensing images in July are selected uniformly in this study ${ }^{[3]}$. In addition, considering such factors as time span, lower cloud cover and high and low quality of remote sensing images, satellite images of 1989, 2001 and 2016 were selected. See Table 1 for details of the data. As the study area is small, one scene can be selected every year.

Table 1 Details of Landsat satellite image data

\begin{tabular}{cccccc}
\hline year & date & Type of sensor & The ranks no. & Cloud cover & Spatial resolution \\
\hline 1989 & 7.29 & Landsat 4-5 TM & $123 / 31$ & 0.1 & $30 \mathrm{~m}$ \\
2001 & 7.6 & Landsat 7 ETM SLC_on & $123 / 31$ & 1.54 & $30 \mathrm{~m}$ \\
2016 & 7.7 & Landsat 8 OLI_TIRS & $123 / 31$ & 2.95 & $30 \mathrm{~m}$ \\
\hline
\end{tabular}

\section{Research results and analysis}

In order to facilitate comparative observation and analysis, this paper divided the vegetation coverage map of Saihanba Mechanized Forest Farm into five grades by referring to the classification of vegetation coverage grades in many studies. The results are shown in Figure 1, Figure 2 and Figure 3. It can be seen from Figure 1 that in 1989, the western part of Saihanba Mechanical Forest Farm had a large area of extremely high vegetation coverage, but also a small area of medium and high vegetation coverage; the central part had medium and medium low vegetation coverage, while the eastern part was dominated by very low vegetation coverage. It can be seen from Figure 2 that in 2001, there was a large area of extremely high vegetation cover in the west of Saihanba mechanical forest farm, and the central area was mainly distributed in the areas of extremely high, medium and medium low vegetation cover, while the eastern area was still very low vegetation cover and a small part of medium and low vegetation cover. As can be seen from Figure 3, Saihanba Mechanical Forest Farm in 2016 was a very high vegetation coverage area on the whole, with very low vegetation coverage scattered.

From 1989 to 2001, the vegetation coverage of Saihanba mechanical forest farm was in a slow rising state, and the change was not very obvious. The vegetation coverage increased mainly in the western and central regions, while the vegetation coverage in the eastern region was still very low, which may be caused by low survival rate at the early stage of vegetation planting, incomplete improvement of soil conditions and immature planting technology. From 2001 to 2016, vegetation situation of sihanba mechanical forest farm has improved, the vegetation coverage is higher in western areas, vegetation coverage is relatively low in central area and vegetation coverage is very low in the east area were all converted to high vegetation coverage, but there are still sporadic low vegetation coverage, this could be due to the area of rock covering area is not suitable for vegetation growth.

\section{Conclusion and prospect}

\subsection{Conclusion}

In this paper, based on RS dynamic monitoring and analysis of Saihanba mechanical forest farm vegetation coverage, the NDVI value was calculated by using the normalized vegetation index, the vegetation coverage was calculated by using the pixel dichotomy method, and the causes of vegetation coverage change were elucidated.

In a word, Saihanba Mechanical Forest Farm, which is seriously desertification due to over-reclamation, has been greatly improved through the unremitting efforts of three generations of foresters. This effectively prevented the Gobi from approaching Beijing, and the water resources in the Beijing-Tianjin area were conserved. The social and ecological benefits are immeasurable. 


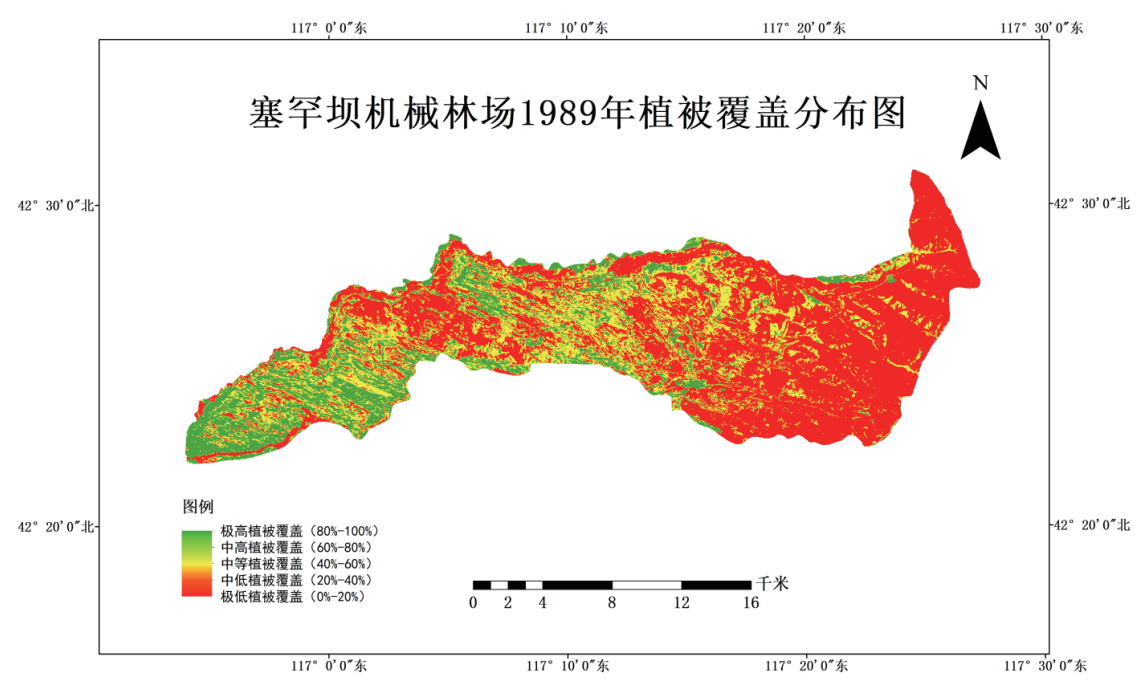

Figure 1. Vegetation coverage distribution in Saihanba Mechanical Forest Farm in 1989

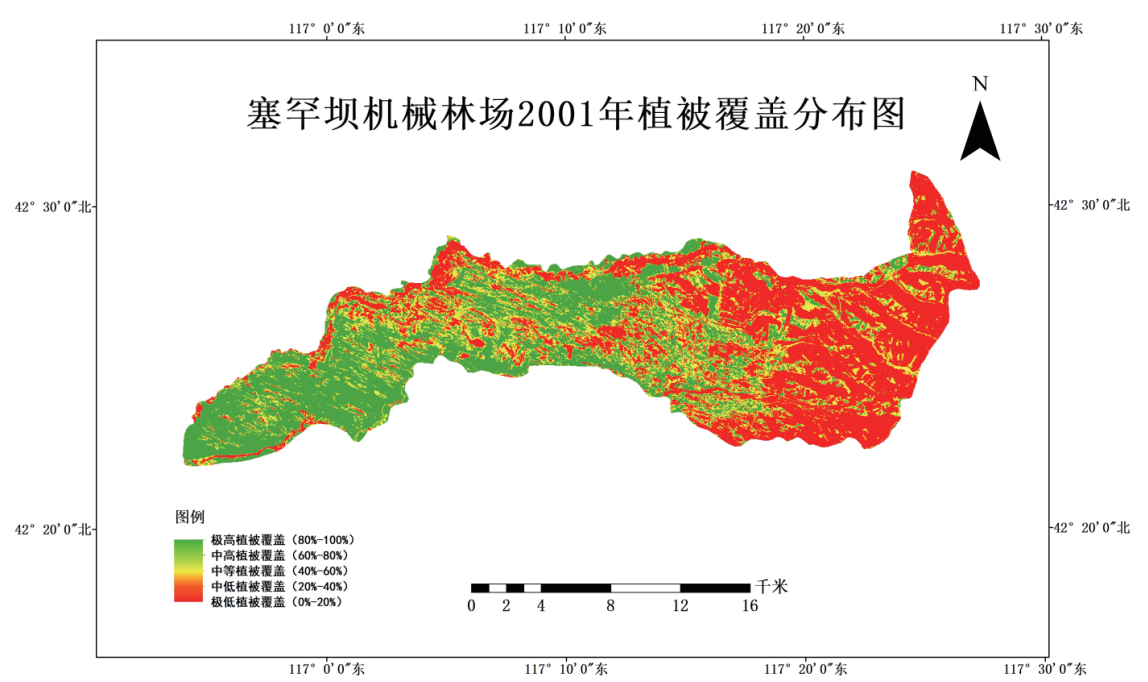

Figure 2. Vegetation coverage distribution map of Saihanba mechanical forest farm in 2001

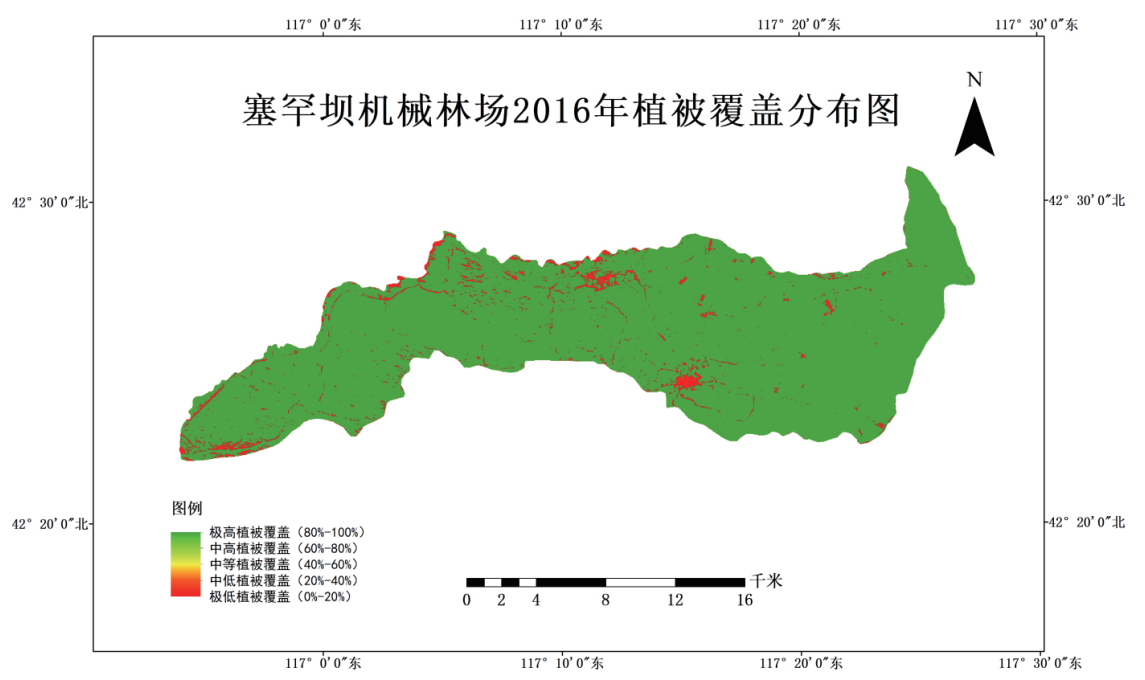

Figure 3. Vegetation coverage distribution map of Saihanba Mechanical Forest Farm in 2016 


\title{
Study on the Pricing Mechanism of Deductive Right License Transfer Based on Mechanism Design Theory
}

\author{
Ting Tang* \\ Shaanxi Provincial Geological Survey Institute, Xi'an 710000, China.
}

\begin{abstract}
The relevant laws and regulations in our country only provide in principle for the license transfer of deductive right. In practice, we often encounter two major problems: First, there is information asymmetry between the original author and the deductive author, and there is a large transaction cost. The second is how to give consideration to the interests of the original author and the deductive author to achieve incentive compatibility. To solve these two problems, build a quantitative model. It has a certain reference value to the practice of deduction right license transfer.

Keywords: Deductive Weight; Economic Analysis; Pricing Mechanism Design
\end{abstract}

\section{Problem presentation}

The deductive work is based on the original work, but rather than simply copying the original work, it is a new work produced after a certain transformation of the original work ${ }^{[1-4]}$. Although China's Copyright Law, the Measures for the Payment of Remuneration for Use of Written Works and other laws and regulations provide relevant provisions on the licensing and transfer of copyright. It also deals with the amount and the method of payment, but the regulation is relatively macroscopic, and the operability is not strong. The low transfer cost is not conducive to the protection of the legitimate rights and interests of the original author, and the high transfer cost hinders the promotion of the project.

It mainly focuses on the relationship between the deductive works and the original works, the definition of the scope of deductive works and whether the legitimate rights and interests of illegal deductive works should be protected. It's more about theoretical issues. However, there are few studies on specific issues such as value orientation, transfer mode, payment standard and calculation of transfer cost of copyright transfer ${ }^{[5-7]}$. In fact, deductive right is a contractual relationship between the original author and the author of the licensing of deductive right. In view of this, this study analyzes the economics studies in mechanism design with the concept of the theory of mechanism design theory, then the deductive works using mechanism design theory to study the legal license fee pricing mechanism and analyze its feasibility and applicability; on this basis, establish reasonable assumptions, build the deductive works licensing cost model for the quantitative study of pricing mechanism.

Copyright $(C 2020$ Ting Tang

doi: $10.18282 / 1-$ e.v9i4.1715

This is an open-access article distributed under the terms of the Creative Commons Attribution Non-Commercial License (http://creativecommons.org/licenses/by-nc/4.0/), which permits unrestricted non-commercial use, distribution, and reproduction in any medium, provided the original work is properly cited.

\subsection{Prospect}

Due to time and energy constraints, FVC calculation and difference calculation were not carried out for each year from 1989 to 2016, but remote sensing images in 1989, 2001 and 2016 were selected for rough dynamic change analysis. Failing to analyze the turning years of vegetation coverage, the dynamic change shows more general.

In the following research, the vegetation cover map of July of each year from 1989 to 2016 will be superimposed by space operation to obtain the area transfer matrix of adjacent years, so as to more intuitively analyze the vegetation cover change in the study area. In short, more accurate data, newer technologies and more perfect methods are adopted to study vegetation cover, so as to obtain more reliable dynamic change data of vegetation cover.

\section{References}

[1] Li Dekun; Cao Ming (2019). Rising Green Miracle in the Wasteland -- Sample Significance of Saihanba Mechanical Forest Farm Construction and Development in More than 50 Years [J]. Lei feng, No. 58, 9-45.

[2] Mi D Y (2018). Analysis of ecological benefit and economic value of Saihanba National Forest Park [J]. Modern Horticulture,No.364, 173.

[3] Liu Jingnan; Ting-bin zhang; Yan-bin qin; Gui-hua yi; Don't xiaojuan [2015]. Dynamic monitoring of vegetation coverage in Wuzhiling mountain area of Henan Province in recent 30 years [J]. Science and Technology Management of Land and Resources,v.32; No. 176, 111-117. 